\title{
Comparison between traditional and nontraditional add-on devices used with pressurised metered-dose inhalers
}

\author{
Mina Nicola ${ }^{1}$, Youssef M.A. Soliman², Raghda Hussein ${ }^{1}$, Haitham Saeed ${ }^{1}$ and \\ Mohamed Abdelrahim (10)
}

Affiliations: ${ }^{1}$ Dept of Clinical Pharmacy, Faculty of Pharmacy, Beni-Suef University, Beni-Suef, Egypt. ${ }^{2}$ Dept of Chest Diseases, Faculty of Medicine, Cairo University, Cairo, Egypt.

Correspondence: Mohamed Abdelrahim, Dept of Clinical Pharmacy, Faculty of Pharmacy, Beni-Suef University, Beni-Suef, 62511, Egypt. E-mail: mohamedemam9dyahoo.com

ABSTRACT Add-on devices that are attached to metered-dose inhalers (MDIs) were introduced to improve aerosol delivery. The objective of this study was to determine the efficacy of drug delivery from an MDI when attached to different add-on devices at different inhalation volumes.

The total emitted dose (TED) of salbutamol was estimated for the MDI alone and the MDI connected to five different add-on devices (Able valved holding chamber, Tips-haler valved holding chamber, Aerochamber plus flow $\mathrm{Vu}$ valved holding chamber, Dolphin chamber, and a handmade water bottle spacer), at inhalation flow of $28.3 \mathrm{~L} \cdot \mathrm{min}^{-1}$ with flow volume of 1,2 and $4 \mathrm{~L}$, assuming young child (aged $<6$ years), old child ( $>6$ years) and adult inhalation volumes, respectively.

The TED\% ranged between $84.1 \%$ and $87.2 \%$ at all inhalation volumes from the MDI alone, which was significantly greater than all MDI add-on device combinations $(\mathrm{p}<0.05)$. The TED\% delivered to MDI sampling apparatus by a homemade water bottle spacer and Dolphin chamber, as non-antistatic add-on devices, ranged between $30.5 \%$ and $35.3 \%$. However, washing these non-antistatic add-on devices with a light detergent before use improved their TED to range between $47.6 \%$ and $51.2 \%$. Non-antistatic add-on devices had significantly lower TED $(\mathrm{p}<0.05)$ than that delivered by most antistatic add-on devices, which ranged from $51.3 \%$ to $71.6 \%$.

This study suggests that antistatic add-on devices delivered much more aerosol than non-antistatic addon devices. However, it may be advised to still use a non-antistatic add-on device, for the sake of solving the coordination problem, and wash it with light detergent before use to improve TED.

@ERSpublications

Antistatic add-on devices deliver much more aerosol from MDIs than non-antistatic add-on devices https://bit.ly/3gSE5aR

Cite this article as: Nicola M, Soliman YMA, Hussein R, et al. Comparison between traditional and nontraditional add-on devices used with pressurised metered-dose inhalers. ERJ Open Res 2020; 6: 00073-2020 [https://doi.org/10.1183/23120541.00073-2020].

This article has been revised according to the author correction published in ERJ Open Res 2021; 7: 50073-2020 [https://doi.org/10.1183/23120541.50073-2020].

Received: 17 Feb 2020 | Accepted after revision: 2 June 2020 | First published: 13 Oct 2020

Copyright $\odot$ ERS 2020. This article is open access and distributed under the terms of the Creative Commons Attribution Non-Commercial Licence 4.0. 


\section{Introduction}

Although systemic delivered therapy is available for asthma and chronic obstructive pulmonary disease (COPD), inhaled therapy is the most widely used option [1-3]. The reason behind that is the better safety index for the inhaled way [4-6]. Metered-dose inhalers (MDIs) are the most commonly used devices for aerosol delivery to the airways. However, most patients, especially the elderly and children do not use it correctly by committing a poor inhalation technique. In other words, a slow inspiration (around $30 \mathrm{~L} \cdot \mathrm{min}^{-1}$ ) should be done by the patient while actuating the MDI (hand-breath coordination) [7, 8]. Also, other errors of technique include not shaking the MDI before use, fast inspiration, not sealing the lips on mouthpiece of the MDI before use and abrupt stopping of inspiration when the MDI plume hits the pharynx (cold freon effect) which is much less with hydrofluoroalkanes MDIs than with chlorofluorocarbons [5, 7, 9]. Moreover, when using MDIs, at least $50-80 \%$ of the drug deposits in the mouth and the throat with only a very small amount (10-15\%) of the drug reaching the airways [10].

Hence, placing a reservoir device (spacer and a valved holding chamber between the MDI and the patient's mouth gives more time for drug inhalation as it gives more volume and space for the propellant to evaporate $[11,12]$. In other meaning, these add-on devices make large particles, emitted by the MDI, deposit within it rather than being deposited in the mouth and throat. Also, the extra distance travelled by the aerosol within the add-on devices increases the dose of fine particles $(<5 \mu \mathrm{m})$ which is the optimal particle size that can reach the deep smaller airways $[12,13]$.

Electrostatic charge is an important key factor that affects the performance of the add-on devices [14]. Most available add-on devices are made of cheap, non-conducting material that accumulates static charge over time (non-antistatic add-on devices) [14, 15]. Thus, innovations have been made to solve this problem by developing antistatic add-on devices, which were patented in 1991; however, they cost more than the older non-antistatic add-on devices, which can be an issue. especially in poorer and developing countries $[14,16]$

Spacers/valved holding chambers with a facemask rather than a mouthpiece may be preferable for children and for those patients incapable, for any reason, of making a slow inspiratory effort while holding a mouthpiece between the teeth [17]. However, spacers/valved holding chambers with facemasks designed for use with young children may provide a poor seal with the face, resulting in impaired or variable dosage delivery which may be exacerbated in young or uncooperative patients. Thus, a tight but comfortable facemask-face seal using sufficient force is critical $[18,19]$.

Disadvantages related to the commercial add-on devices include additional expense and the requirement of a prescription, costing roughly 15-40 USD [20]. Moreover, these devices are also difficult to obtain in a developing country $[20,21]$. Thus, the use of nontraditional add-on devices such as a plastic drink bottle spacer or other suitable materials could be cost-saving without sacrificing clinical effectiveness [22-24].

The present study aimed to evaluate the efficacy of a range of add-on devices with and without a facemask in delivering the aerosolised drugs from an MDI at different inhalation volumes, 4, 2 and $1 \mathrm{~L}$ assuming adult, old child (aged $>6$ years), and young child ( $<6$ years) inhalation volumes, respectively.

\section{Methods}

The emitted dose from a Ventolin MDI (labelled as a nominal dose of $100 \mu \mathrm{g}$ salbutamol per puff; GlaxoSmithKline, Cairo, Egypt) was determined. Determinations were made for the MDI and when the MDI was attached to different add-on devices. The add-on devices were the Able valved holding chamber, $208 \mathrm{~mL}$, and antistatic (Clement Clarke International Ltd, Harlow, UK), Aerochamber plus flow Vu holding chamber, $149 \mathrm{~mL}$, and antistatic (Trudell Medical International Europe Ltd, Basingstoke, UK), Dolphin chamber, $133 \mathrm{~mL}$, non-antistatic (TMS Medikal Plastik Sanayi ve Dış Ticaret Ltd. Şti., Istanbul, Turkey), Tips-haler holding chamber, $260 \mathrm{~mL}$, and antistatic (Laboratoire ProtecSom-OptimHal, Valognes, France) and a spacer made from a water bottle, $600 \mathrm{~mL}$ (Dasani bottle; The Coca-Cola Company, Cairo, Egypt).

We used each add-on device with its availability in the market, with facemask and mouthpiece, in the three tested inhalation volumes as shown in figure 1. Child masks, if present, for lower volume and adult mask for higher volume. If the mask is one size we used it in the tested volumes. We used a homemade spacer with the only mouthpiece.

Non-antistatic devices were washed with detergent and left to dry in air before being retested for aerosol delivery.

The MDI sampling apparatus (Copley Scientific Ltd, UK) with a critical flow controller model TPK (Copley Scientific Ltd, UK) was used. The final filter was a $25-\mathrm{mm} \mathrm{A/E} \mathrm{fibreglass} \mathrm{filter} \mathrm{(Pall} \mathrm{Corporation,}$ USA). Vacuum flow through the apparatus was provided by a Vacuum pump (Brook Crompton, UK). 


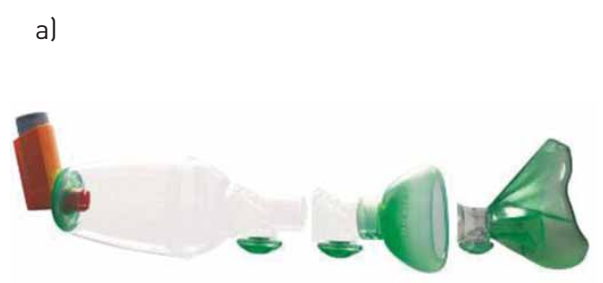

b)

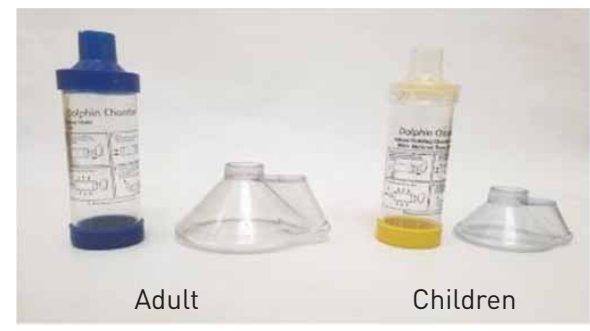

c)

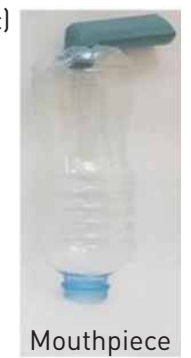

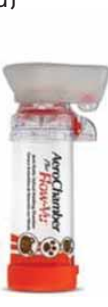

Small

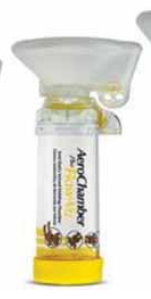

Medium

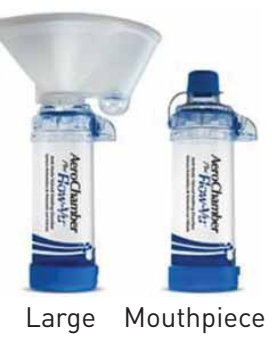

e)

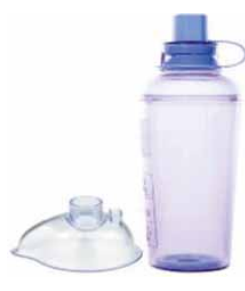

FIGURE 1 The five devices and valved holding chambers used in the study: al Tips-haler spacer; b) Dolphin chamber; c) homemade spacer from a water bottle; d) Aerochamber plus flow Vu chamber; e) Able spacer.

Inhalation flow was set at $28.3 \mathrm{~L} \cdot \mathrm{min}^{-1}$ over durations of around 8.5, 4.25 and $2.13 \mathrm{~s}$ providing inhalation volumes equal to 4, 2 and $1 \mathrm{~L}$ respectively for each device, estimated to simulate the breathing of adults, old children and young children, respectively, using mouthpieces and facemasks. Flow was measured by an electronic digital flow meter (MKS Instruments, USA), and Parafilm M, laboratory film (Pechiney Plastic Packaging, USA), was used to seal the apparatus.

MDI testing setting is shown in figure 2a. Each MDI/add-on device mouthpiece was connected tightly to the mouthpiece adaptor of the dose-sampling unit and aligned along the horizontal axis as shown in figure $2 \mathrm{~b}$. In the case of facemask interface, a plate was added to the experimental setup and the facemask was sealed to it as shown in figure $2 \mathrm{c}$. This plating method was previously shown to have no significant difference to the face method for the determination of the inhalable mass from different aerosol generators
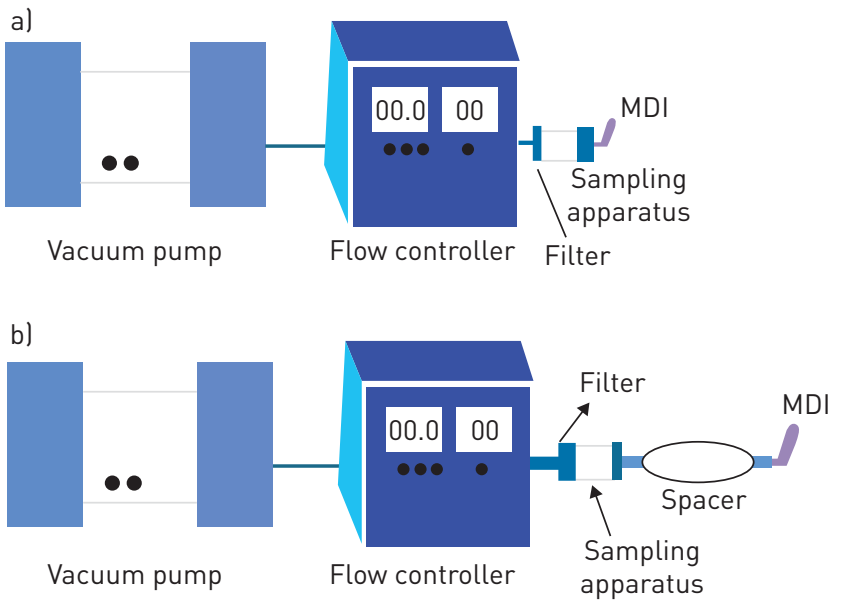

FIGURE 2 Schematic diagram illustrating the measurement of total inhalable dose using a) metered-dose inhaler (MDI) alone, b) MDI with mouthpiece spacer and c) MDI with facemask spacer.

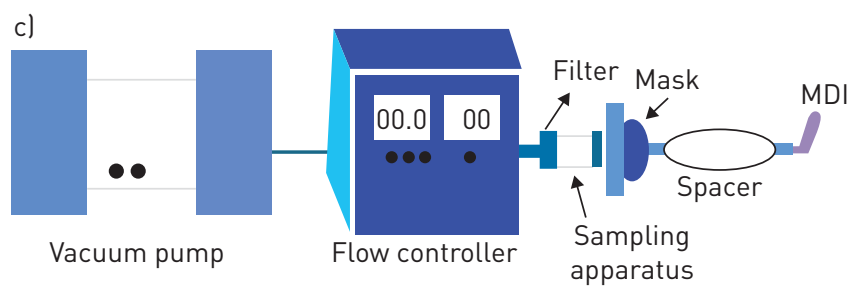


with facemask $[18,25,26]$. The emitted dose from the MDI and MDI+add-on device was measured by collecting one individual dose at $28.3 \mathrm{~L} \cdot \mathrm{min}^{-1}$ with an inhaled volume of 4,2 and $1 \mathrm{~L}$. 10 determinations were made for each dose emission $(n=10)$. The MDI was shaken and primed by firing two doses to waste before use [27]. The discharge of the dose (pressing the canister) was done in coordination (at the same time) with the switching on of the pump in case of the MDI without an add-on device. When using the add-on device, the dose was discharged into the add-on device and with the switching on of the pump within $1 \mathrm{~s}$.

Following dose emission into the apparatus, the add-on device, the sampling unit was washed with $90 \%$ acetonitrile and the filter was completely submerged in $90 \%$ acetonitrile and then sonicated for $3 \mathrm{~min}$ (preliminary analysis revealed that this procedure removes all drug entrained on the filter) [28, 29]. The amount of drug was determined by high-performance liquid chromatography using the previously validated method [28]. The total emitted dose (TED) was the amount deposited in the plastic dose sampling apparatus and the final filter.

Salbutamol was quantified with high-performance liquid chromatography linked to an ultraviolet detector. Samples of the acetonitrile washings $(100 \mu \mathrm{L})$ were eluted on a $25 \times 4.6 \mathrm{~mm}$ ZORBAX Eclipse Plus C18, ODS1 column (Agilent, Santa Clara, CA, USA) using a 90/10 (v/v) acetonitrile and water mobile phase that also contained $0.1 \%$ phosphoric acid pumped at a flow of $1 \mathrm{~mL} \cdot \mathrm{min}^{-1}$ (1260 Infinity preparative pump, G1361A, Agilent). The detector (1260 Infinity Diode array detector VL, G131SD, Agilent) was set at $225 \mathrm{~nm}$ and calibration was performed using solutions ranging from 4 to $100 \mu \mathrm{g} \cdot \mathrm{mL}^{-1}(\mathrm{w} / \mathrm{v})$. The limit of detection used was $0.3 \mathrm{ug} \cdot \mathrm{mL}^{-1}$, and the lower limit of quantification was $2.5 \mu \mathrm{g} \cdot \mathrm{mL}^{-1}[28,29]$.

\section{Statistical analysis}

Comparison of the TED for the MDI alone and the different MDI/add-on device combinations was made using a two-way ANOVA testing using SPSS software 23 (SPSS, Chicago, IL, USA).

\section{Results}

The mean \pm SD TED of salbutamol and dose deposited in the add-on devices are shown in tables 1-3. The amounts are expressed as a percentage of the $100 \mu \mathrm{g}$ salbutamol nominal dose. The highest value observed was the TED from the MDI and was significantly higher than all MDI/add-on device combinations $(\mathrm{p}<0.05)$ with all inhalation volumes performed in the study $(4,2$ and $1 \mathrm{~L})$.

For the mouthpiece and facemask combinations of MDI add-on device; the MDI-Dolphin chamber and MDI-handmade drink bottle spacer in $4 \mathrm{~L}$ and $2 \mathrm{~L}$ significantly showed the lowest TED than any antistatic add-on device $(\mathrm{p}<0.05)$. However, no significant $(\mathrm{p}=0.074)$ difference was found between the TED delivered from the Dolphin chamber and handmade drink bottle spacer.

The mean TED results from the MDI-Tips-haler facemask and mouthpiece combinations in all inhalation volumes were lower than their corresponding mask and mouthpieces of MDI-Able and MDI-Aerochamber plus flow $\mathrm{Vu}$; however, the difference was not significant $(\mathrm{p}=0.068)$.

TABLE 1 Outcome of salbutamol dose delivered via metered-dose inhaler (MDI) and different $\mathrm{MDI}$-spacer combinations in $4 \mathrm{~L}$ volume of inhalation (assuming adult inhalation volume)

\begin{tabular}{lccc} 
MDI or MDI/accessory device & Nature & Emitted dose \% & Dose deposited in spacer \% \\
\hline MDI only & & $86.8 \pm 5.1$ & $\mathrm{NA}$ \\
Aerochamber mouthpiece & Antistatic & $71.6 \pm 4.4$ & $16.8 \pm 3.30$ \\
Aerochamber mask & Antistatic & $69.7 \pm 5.4$ & $18.9 \pm 4.9$ \\
Able spacer mouthpiece & Antistatic & $64.2 \pm 6.9$ & $23.2 \pm 4.3$ \\
Able spacer mask & Antistatic & $61.2 \pm 6.6$ & $24.1 \pm 4.9$ \\
Tips-haler mouthpiece & Antistatic & $53.6 \pm 7.2$ & $34.4 \pm 7.4$ \\
Tips-haler mask & Antistatic & $51.3 \pm 4.7$ & $34.6 \pm 5.8$ \\
Dolphin chamber mouthpiece & Non-antistatic & $31.9 \pm 5.4$ & $56 \pm 4.7$ \\
Dolphin chamber mask & Non-antistatic & $30.5 \pm 5.6$ & $\mathrm{NA}$ \\
Dolphin chamber wash & Non-antistatic & $49.7 \pm 6.4$ & $50.2 \pm 4.3$ \\
Drink bottle mouthpiece & Non-antistatic & $35.3 \pm 6.9$ & $\mathrm{NA}$ \\
Drink bottle wash & Non-antistatic & $51.2 \pm 7.1$ & \\
\hline Data are presented as mean \pm SD. NA: not applicable. & & \\
\hline
\end{tabular}


TABLE 2 Outcome of salbutamol dose delivered via metered-dose inhaler (MDI) and different MDI-spacer combinations in $2 \mathrm{~L}$ volume of inhalation lassuming old child inhalation volume)

\begin{tabular}{lccc} 
MDI or MDI/accessory device & Nature & Emitted dose \% & Dose deposited in spacer \% \\
\hline MDI only & & $87.2 \pm 6.9$ & $\mathrm{NA}$ \\
Aerochamber mask & Antistatic & $69.8 \pm 5.8$ & $18.7 \pm 4.1$ \\
Able spacer mouthpiece & Antistatic & $66.2 \pm 6$ & $22.3 \pm 4.3$ \\
Able spacer mask & Antistatic & $64.3 \pm 5.1$ & $24.1 \pm 4.9$ \\
Tips-haler mouthpiece & Antistatic & $53.9 \pm 6.5$ & $38.2 \pm 4.5$ \\
Tips-haler mask & Antistatic & $51.5 \pm 6.1$ & $34.5 \pm 6$ \\
Dolphin chamber mouthpiece & Non-antistatic & $32.4 \pm 6.7$ & $53.7 \pm 5.5$ \\
Dolphin chamber mask & Non-antistatic & $32.2 \pm 6.4$ & $57.6 \pm 3.5$ \\
Dolphin chamber wash & Non-antistatic & $47.6 \pm 5.9$ & $\mathrm{NA}$ \\
Drink bottle mouthpiece & Non-antistatic & $34.3 \pm 7.3$ & $\mathrm{NA}$ \\
Drink bottle wash & Non-antistatic & $49.9 \pm 6.2$ & \\
\hline Data are presented as mean \pm SD. NA: not applicable. & &
\end{tabular}

Concerning the mean drug fraction accumulated on the add-on device walls, the MDI-Tips-haler of the facemask and mouthpiece were higher than their corresponding values from the combinations of MDI with Able device and Aerochamber plus flow $\mathrm{Vu}$; however, the difference was not significant; however, the handmade bottle spacers and the dolphin chamber in all volume of inhalations resulted in higher mean drug deposition on the add-on device walls compared with the antistatic add-on devices $(\mathrm{p}<0.05)$.

Washing non-antistatic devices and letting them dry in the air has a significant impact on the delivered doses from the Dolphin chamber and drink bottle; however, their TED was still lower than that from anti-static devices.

No significant difference was found in terms of facemask and mouthpieces comparison in all types of add-on devices tested at the same volume of inhalation appointed (comparison within the same type of add-on device).

\section{Discussion}

The TED released from the MDI alone was the highest dose compared with that released from the other combinations of MDI and add-on devices. The greatest delivered dose emitted from the MDI alone was expected due to the absence of an attached add-on device, which reduces the number of large aerosol particles reaching the MDI sampling unit, expected to deposit in the mouth [30]. When using MDI alone, at least $50-80 \%$ of the drug deposits in the mouth and the throat with only a very small amount of the drug reaching the airways [10].

The main finding presented in this study has revealed that MDI-add-on devices with antistatic nature (Aerochamber plus flow Vu chamber, Tips-haler spacer, and Able spacer) made the best (highest) TED and the lowest drug deposition on the add-on device walls compared to all other non-antistatic add-on devices (Dolphin chamber and handmade bottle drink spacer) $[14,15,19,20]$. The reason beyond this result might be attributed to the non-ideal design and the static charge found on the add-on device walls $[14,19]$. However, non-antistatic devices might help in certain situations like the lack of coordination and emergency condition with the unavailability of the antistatic spacers, especially in developing countries.

TABLE 3 Outcome of salbutamol dose delivered via metered-dose inhaler (MDI) and different MDI-spacer combinations in $1 \mathrm{~L}$ volume of inhalation lassuming young child inhalation volume)

\begin{tabular}{lccc} 
MDI or MDI/accessory device & Nature & Emitted dose \% & Dose deposited in spacer \% \\
\hline MDI only & & $84.1 \pm 6$ & NA \\
Aerochamber mask & Antistatic & $58.3 \pm 6.5$ & $30.1 \pm 6.9$ \\
Able spacer mask & Antistatic & $55.6 \pm 6.3$ & $31.6 \pm 4.2$ \\
Tips-haler mask & Antistatic & $46.4 \pm 5.8$ & $38.3 \pm 9.8$ \\
\hline \multicolumn{2}{l}{ Data are presented as mean \pm SD. NA: not applicable. } \\
\hline
\end{tabular}


Washing non-antistatic devices and letting them dry in the air has a significant impact on the delivered doses from the Dolphin chamber and drink bottle. This result could be explained by the neutralising effect of detergent (antistatic nature) that decreased the deposition of aerosol particles and allowed the delivery of higher amounts. The main drawback related to the use of detergent is the time needed for the devices to dry, which is of particular importance in emergencies.

The superiority showed in our results of antistatic add-on devices over the non-antistatic add-on devices could be related to the antistatic nature, shape, volume and presence of a valve.

Although the Tips-haler has an antistatic nature and a valve, other antistatic add-on devices (the Aerochamber plus flow $\mathrm{Vu}$ chamber and Able valved holding chamber) have revealed a nonsignificant higher mean TED beside lower significant drug deposition inside them than the Tips-haler spacer $(p<0.05)$. These findings should pose a question; if Tips-haler nonsignificant lower results are attributed to Tips-haler removal of large drug particles of nontherapeutic value. This question can be answered by extending our study to an in vivo setting in order to detect this effect.

As it is well known that a tight (but comfortable) facemask-to-face seal using sufficient force is important to achieve patient tolerance and avoid leakage of the drug out the system, the more force applied to the facemask on the face the tighter the seal; thus, this could be what leads the patients to favour an elastic facemask over a non-elastic one [18, 19].

There was only a weak relative (nonsignificant) TED difference in favour of mouthpieces over full facemasks. This insignificant difference might be related to the tight seal applied in our in vitro model.

Delivering an aerosolised drug to a child is a complex technique that requires the involvement of parents, the child and the inhalation device. Previous studies have revealed that the facemask is a key factor affecting the delivery of aerosols, especially due to leaks exacerbated by the screaming and movement of children and non-tolerance, as well as poor hand strength in adults using these facemasks [18, 31]. These are absent in our in vitro model; hence, extra in vivo work is required to test the process of poor seal and leaks, which is essentially absent due to the robust pressure applied in this study.

We found that the effect of antistatic add-on devices in delivering the aerosolised particles was about double $(\mathrm{p}<0.05)$ of that observed in the nontraditional handmade drinking bottle spacer. Furthermore, the effect of the drinking bottle spacer was similar (nonsignificant difference) to the traditional non-antistatic marketed add-on device. An essential use for add-on devices could be to provide inhaled drugs efficiently in an emergency (such as an acute exacerbation of pulmonary obstructive disease). As an emergency can occur anywhere and at any moment, it could be difficult to find and provide expensive commercial add-on devices for such situations [23]. Hence, using a drinking bottle spacer, while doubling the initial dose, would be helpful and cost effective, especially in poor and developing communities [20]. Therefore, further in vivo investigations and comparisons between traditional and nontraditional MDI add-on devices should be made in the future to illustrate and confirm the results of this in vitro study.

\section{Limitations of the study}

Although this in vitro study was effective regarding making a comparison between the MDI and the different add-on devices, the study lacked estimation of an in vivo clinical response and tolerance in patients.

Another limitation was that the add-on devices in this study were not all the commercially available devices with other different shapes and materials which may yield other different outcomes.

The study had some unrealistic results since a young child to inhale a $1 \mathrm{~L}$ volume at 28.3 would be in $2.13 \mathrm{~s}$ which is hard to be constant since it is hard to make the patient inhale at the proper inhalation flow. That is why we are extending this work to in vivo to compare children to adults with and without different spacers.

\section{Conclusions}

This investigation suggests that the antistatic nature of the antistatic add-on devices (Aerochamber plus flow Vu, Tips-haler, and Able valved holding chambers) made them the best choices over any non-antistatic add-on devices examined here in terms of delivered aerosol. Even though the homemade drink bottle spacer and Dolphin chamber did not significantly improve TED, they might improve synchronisation between actuation and inhalation.

No significant difference was found in terms of facemask and mouthpieces comparison in all types of devices tested at the same volume of inhalation appointed. 
Author contributions: M. Nicola performed the clinical study and wrote the manuscript. Y.M.A. Soliman and R. Hussein conceived and planned the study, and wrote the manuscript. H. Saeed performed data entry, wrote the manuscript and performed statistical analysis. M. Abdelrahim conceived and planned the study, and wrote the manuscript.

Conflict of interest: None declared.

\section{References}

1 Nicola M, Elberry A, Sayed $\mathrm{O}$, et al. The impact of adding a training device to familiar counselling on inhalation technique and pulmonary function of asthmatics. Adv Ther 2018; 35: 1049-1058.

2 Nicola M, Elberry AA, Sayed OM, et al. Effect of DPI's training-device on inhalation technique and clinical efficacy in asthmatics. Beni-Suef Univ J Basic Appl Sci 2018; 7: 178-183.

3 Elgendy MO, Abdelrahim ME, Eldin RS. Potential benefit of repeated dry powder inhaler's inhalation technique counseling on asthmatic patients. Pulm Ther 2015; 1: 91-101.

4 Willemse B, Toelle BG, Li JS, et al. Use of a paper disposable cup as a spacer is effective for the first-aid management of asthma. Respir Med 2003; 97: 86-89.

5 Saeed H, Salem HF, Rabea H, et al. Effect of human error, inhalation flow, and inhalation volume on dose delivery from Ellipta dry-powder inhaler. J Pharm Innov 2019; 14: 239-244.

6 Elgendy MO, Abdelrahim ME, Eldin RS. Potential benefit of repeated MDI inhalation technique counselling for patients with asthma. Eur J Hosp Pharm 2015; 22: 318-322.

7 McFadden E Jr. Improper patient techniques with metered dose inhalers: clinical consequences and solutions to misuse. J Allergy Clin Immunol 1995; 96: 278-283.

8 Dolovich MB, Ahrens RC, Hess DR, et al. Device selection and outcomes of aerosol therapy: evidence-based guidelines: American College of Chest Physicians/American College of Asthma, Allergy, and Immunology. Chest 2005; 127: 335-371.

9 Saeed H, Abdelrahim ME, Rabea $\mathrm{H}$, et al. Impact of advanced patient counseling using a training device and smartphone application on asthma control. Respir Care 2020; 65: 326-332.

10 National Asthma Education. Expert panel report 2: guidelines for the diagnosis and management of asthma. Darby, Diane Publishing, 1998.

11 Kwok PCL, Collins R, Chan H-K. Effect of spacers on the electrostatic charge properties of metered dose inhaler aerosols. J Aerosol Sci 2006; 37: 1671-1682.

12 Rau JL. Practical problems with aerosol therapy in COPD. Respir Care 2006; 51: 158-172.

13 Rahmatalla MF, Zuberbuhler PC, Lange CF, et al. In vitro effect of a holding chamber on the mouth-throat deposition of QVAR (hydrofluoroalkane-beclomethasone dipropionate). J Aerosol Med 2002; 15: 379-385.

14 Anderson G, Johnson N, Mulgirigama A, et al. Use of spacers for patients treated with pressurized metered dose inhalers: focus on the VENTOLIN Mini Spacer. Expert Opin Drug Deliv 2018; 15: 419-430.

15 Mitchell JP, Coppolo DP, Nagel MW. Electrostatics and inhaled medications: influence on delivery via pressurized metered-dose inhalers and add-on devices. Respir Care 2007; 52: 283-300.

16 Nikander K, Nicholls C, Denyer J, et al. The evolution of spacers and valved holding chambers. J Aerosol Med Pulm Drug Deliv 2014; 27: Suppl. 1, S4-S23.

17 Ditcham W, Murdzoska J, Zhang G, et al. Lung deposition of $99 \mathrm{mTc}$-radiolabeled albuterol delivered through a pressurized metered dose inhaler and spacer with facemask or mouthpiece in children with asthma. J Aerosol Med Pulm Drug Deliv 2014; 27: Suppl. 1, S63-S75.

18 Nikander K, Berg E, Smaldone GC. Jet nebulizers versus pressurized metered dose inhalers with valved holding chambers: effects of the facemask on aerosol delivery. J Aerosol Med 2007; 20: Suppl. 1, S46-S58.

19 Vincken W, Levy ML, Scullion J, et al. Spacer devices for inhaled therapy: why use them, and how? ERJ Open Res 2018; 4: 00065-2018.

20 Sheth P, Bertsch MD, Knapp CL, et al. In vitro evaluation of nonconventional accessory devices for pressurized metered-dose inhalers. Ann Allergy Asthma Immunol 2014; 113: 55-62.

21 Zar HJ, Levin ME. Challenges in treating pediatric asthma in developing countries. Paediatr Drugs 2012; 14 353-359.

22 Asmus MJ, Coowanitwong I, Kwon SH, et al. In vitro performance of two common valved holding chambers with a chlorofluorocarbon-free beclomethasone metered-dose inhaler. Pharmacotherapy 2003; 23: 1538-1544.

23 Kissoon N, Teelucksingh S, Blake KV, et al. Plastic bottles as spacers for a pressurized metered-dose inhaler: in vitro characteristics. West Indian Med J 2001; 50: 189-193.

24 Zar H, Brown G, Donson H, et al. Home-made spacers for bronchodilator therapy in children with acute asthma: a randomised trial. Lancet 1999; 354: 979-982.

25 Madney YM, Laz NI, Elberry AA, et al. The influence of changing interfaces on aerosol delivery within high flow oxygen setting in adults: an in-vitro study. J Drug Deliv Sci Technol 2020; 55: 101365.

26 Vecellio L, Abdelrahim ME, Montharu J, et al. Disposable versus reusable jet nebulizers for cystic fibrosis treatment with tobramycin. J Cyst Fibros 2011; 10: 86-92.

27 Barry PW, O'Callaghan C. The influence of inhaler selection on efficacy of asthma therapies. Adv Drug Deliv Rev 2003; 55: 879-923.

28 Hassan A, Rabea H, Hussein RR, et al. In-vitro characterization of the aerosolized dose during non-invasive automatic continuous positive airway pressure ventilation. Pulm Ther 2016; 2: 115-126.

29 Saeed H, Mohsen M, Fink JB, et al. Fill volume, humidification and heat effects on aerosol delivery and fugitive emissions during noninvasive ventilation. J Drug Deliv Sci Technol 2017; 39: 372-378.

30 Leung SSY, Chiow ACM, Kwok PCL, et al. Effect of spacers on the bipolar electrostatic charge properties of metered dose inhaler aerosols - a case study with Tilade. J Pharm Sci 2017; 106: 1553-1559.

31 Amirav I, Newhouse MT. Aerosol therapy with valved holding chambers in young children: importance of the facemask seal. Pediatrics 2001; 108: 389-394. 